\title{
Multiple Pulmonary Nodules in an Immunocompetent Adolescent with Infectious Mononucleosis
}

\author{
Praveena Nediyara Bhaskaran ${ }^{1}$, Mammen Puliyel ${ }^{2}$, Melissa Myers ${ }^{3}$ and Nazha Abughali ${ }^{4}$ \\ From Departments of ${ }^{1}$ Pediatrics, ${ }^{2}$ Pediatric Hematology, ${ }^{3}$ Radiology and ${ }^{4}$ Pediatric Infectious Disease, MetroHealth Medical \\ Center, Cleveland, OH 44109, USA.
}

Correspondence to:

Dr Praveena Nediyara Bhaskaran, Department of Pediatrics, MetroHealth Medical Center, Cleveland, OH-44109, USA.pbhaskaran@metrohealth.org Received: October 30, 2016;

Initial review: March 20, 2017, Accepted: November 23, 2017.
Background: Infectious mononucleosis is usually a self-limiting illness, but can be rarely associated with complications. Case characteristics: A 17-year-old boy with Epstein-Barr virus related infectious mononucleosis and cold antibody-mediated autoimmune hemolytic anemia with incidentally noted multiple pulmonary nodules. Observations: Nodules regressed over the next few weeks without specific therapy. Message: Pediatricians need to be aware of this rare clinical presentation of infectious mononucleosis so that further invasive testing can be avoided.

Keywords: Computed tomography, Epstein Barr virus, Lung nodules.
$\mathrm{E}$ pstein Barr Virus (EBV) infection is usually a self-limiting disease. The age of the patient has a profound influence on the clinical manifestations. In infants and young children, it is either asymptomatic or accompanied by mild, nonspecific symptoms. In contrast, approximately $50 \%$ of adolescents and young adults present as infectious mononucleosis (IM) [1].

\section{CASE Report}

A 17-year-old previously healthy boy initially presented to the local pediatrician's office with complaints of fever, malaise, bodyache and decreased appetite of 14 days' duration and headache, sore throat and neck pain of 10 days' duration. His girlfriend was diagnosed with infectious mononucleosis a few weeks ago. Physical examination revealed pharyngeal erythema, a grade $3+$ tonsillar enlargement bilaterally with purulent exudates and bilateral anterior and posterior non tender cervical lymphadenopathy. Laboratory investigations revealed hemoglobin $(\mathrm{Hb}) 150 \mathrm{~g} / \mathrm{L}$, white blood cell (WBC) count $5.1 \times 10^{9} / \mathrm{L}$ with $18 \%$ lymphocytes which included $16 \%$ of atypical lymphocytes, 33\% Neutrophils and $19 \%$ band cells. Monospot test was positive. Rapid streptococcal test of the throat swab was negative. Patient was given a dose of intravenous dexamethasone $5 \mathrm{mg}$ for symptomatic relief and was sent home on 2 days of oral steroids.

On day 18 of illness, the patient presented to our emergency department with multiple episodes of syncope, vomiting, poor oral intake and fever. He was found to be febrile and hypotensive and had a weight loss of $7 \%(79.3 \mathrm{~kg}$ to $73.9 \mathrm{~kg})$. There was no history of bleeding from any site. Investigations revealed hemoglobin $80 \mathrm{~g} / \mathrm{L}$, reticulocyte $6.3 \%$, absolute reticulocyte count $210 \times 10^{9} / \mathrm{L}, \mathrm{LDH} 281 \mathrm{U} / \mathrm{L}$ and elevated liver enzymes. Total bilirubin and haptoglobin were normal, and urine hemosiderin was negative. Computed Tomography scan (CT) of abdomen and pelvis did not show any evidence of splenic rupture or hemoperitoneum, but showed moderate hepatosplenomegaly and retroperitoneal lymph node enlargement with the largest one measuring $2.5 \times 2.2 \mathrm{~cm}$. It also revealed multiple pulmonary nodules in the visualized lower lobes with the largest measuring $9 \times 11 \mathrm{~mm}$. CT chest showed about 15-20 pulmonary nodules involving all lobes. The nodules were solid and well circumscribed with spiculated margins.

Direct antiglobulin test was positive for complement and negative for IgG. Cold agglutinins were negative and Donath Landsteiner antibody was negative. The patient received one unit of packed red cell transfusion with an inline warmer. Respiratory viral panel as well as other infectious disease work up for Human Immunodeficiency Virus (HIV), Cytomegalovirus (CMV), Parvovirus and Mycoplasma were negative. Epstein Barr virus Viral Capsid Antigen (EBV-VCA) IgM was positive suggestive of an acute EBV infection. The anemia was attributed to cold antibody related hemolytic anemia due to $\mathrm{EBV}$ infection.

Patient was followed up weekly for the next six 
weeks. After six weeks, his hemoglobin was $111 \mathrm{~g} / \mathrm{L}$. He was asymptomatic at the visit and had regained his weight. Low dose follow-up CT chest two months after the initial presentation showed resolution of most of the nodules.

\section{Discussion}

Infectious mononucleosis can sometimes cause multisystemic involvement including hematologic manifestations, splenic rupture, hepatic involvement and rarely renal, cardiac and pulmonary manifestations. The pulmonary involvement previously reported involved either interstitial or parenchymal pneumonia. In immunosuppressed patients, EBV can also cause lymphoproliferative disorder (PTLD) or lymphoma, which can present as multiple pulmonary nodules [1]. Pulmonary nodules occurring in immunocompetent children as part of EBV is rarely reported in literature. Pelliccia, et al. [2] described a 14-year-old girl with EBV positive IMN with multiple pulmonary nodules as well as mild pericardial effusion and hydrops of gall bladder, all of which resolved on follow up. Shinozuka, et al. [3] described a case of multiple lung nodules in an adolescent with IMN which spontaneously regressed over a two year period.

There has been an increased incidence of detection of pulmonary nodules in children due to widespread use of CT scans. Currently there are no evidence based guidelines for the management of pulmonary nodules in children. Metastatic disease is much more likely to be a cause of a malignant nodule in a child than is a primary lung tumor. The characteristics most likely to be associated with malignancy include nodules with sharp borders, presence of a solid mass with a hilic pattern of growth, or a mixed solid- cystic or completely cystic mass. Fat, popcorn calcification, uniform calcification, peripheral location, elongated lesion, pleural tag and stability compared with prior studies are all associated with benign lesions. [4]

Pulmonary nodules could be part of the clinical presentation of infectious mononucleosis in otherwise healthy children and in an appropriate setting, a close follow-up with complete resolution is adequate to rule out malignancy. Our patient was managed conservatively with close monitoring and regular follow-up. The decision on follow up imaging should be individualized

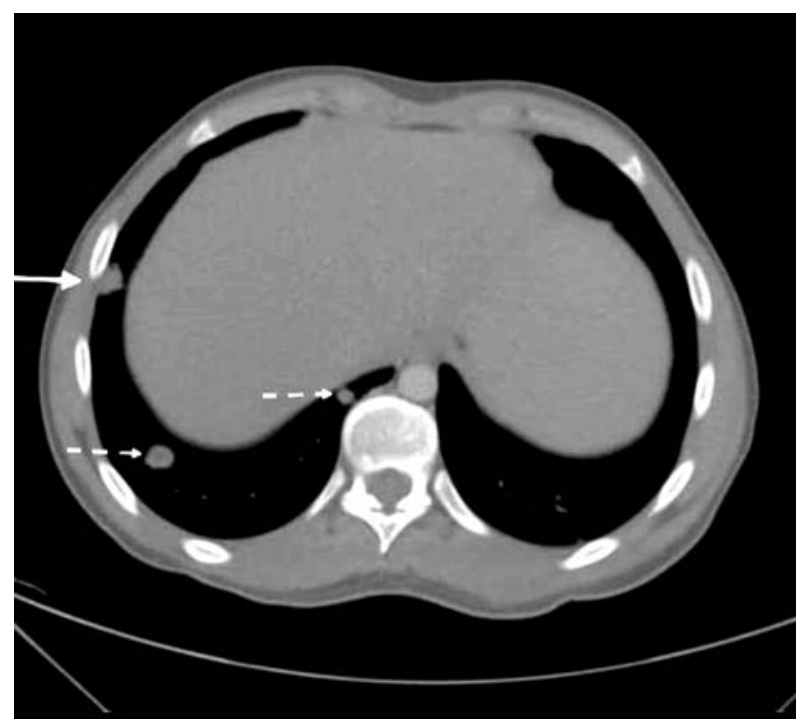

FIG. 1 Axial image through the lung bases showing the multiple pulmonary nodules (broken arrow). The largest one in this image (solid arrow) measures $13.5 \times 1.7 \mathrm{~mm}$.

based on clinical signs and symptoms and imaging characteristics.

Contributors: PNB: drafted the manuscript; MP: contributions to the conception and design of the work and diagnosis of the disease; MM and NA: Revised the work critically for important intellectual content.

Funding: None; Competing interests: None stated.

\section{REFERENCES}

1. Allen C, Rooney CM, Gottschalk S. Infectious Mononucleosis and other Epstein-Barr Virus-associated Diseases. In: Hoffman R, Benz EJ, Silberstein LE, Heslop HE, Weitz JI, Anastasi J, editors. Hematology: Basic Principles and Practice. 6th ed. Philadelphia: Elsevier Inc. 2013. p. 708-20.

2. Pelliccia P, Savino A, Cecamore C, Di Marzio D, Chiarelli $\mathrm{F}$, Primavera A, et al. Imaging spectrum of EBV-infection in a young patient. J Ultrasound. 2008;11:82-4.

3. Shinozuka J, Awaguni H, Tanaka SI, Makino S, Maruyama $\mathrm{R}$, Inaba $\mathrm{T}$, et al. Spontaneous regression of pulmonary nodules presenting as epstein-barr virus-related atypical infectious mononucleosis. J Pediatr Hematol Oncol. 2016;38:e162-5.

4. Brody AS, Mahani MG, Guillerman RP, Hegde SV, Iyer $\mathrm{RS}$, Lee EY, et al. The incidental pulmonary nodule in a child. Part 1: Recommendations from the SPR thoracic imaging committee regarding characterization, significance and follow-up. Pediatr Radiol. 2015;45:628-33. 